\title{
A Brief Review of Historical Supernovae
}

\author{
Shawqi Al Dallal', Walid J. Azzam² \\ ${ }^{1}$ College of Graduate Studies and Research, Ahlia University, Manama, Bahrain \\ ${ }^{2}$ Department of Physics, College of Science, University of Bahrain, Sakhir, Bahrain \\ Email:wjazzam@uob.edu.bh,wjazzam@gmail.com
}

How to cite this paper: Al Dallal, S. and Azzam, W.J. (2021) A Brief Review of Historical Supernovae. International Journal of Astronomy and Astrophysics, 11, 73-86. https://doi.org/10.4236/ijaa.2021.111005

Received: January 21, 2021

Accepted: March 6, 2021

Published: March 9, 2021

Copyright (c) 2021 by author(s) and Scientific Research Publishing Inc. This work is licensed under the Creative Commons Attribution International License (CC BY 4.0).

http://creativecommons.org/licenses/by/4.0/

\begin{abstract}
Supernovae are powerful explosions of massive stars that have reached a terminal stage in their evolution. A huge amount of energy is released during the explosion in a wide range of wavelengths. The supernova explosion causes a sudden rise in the dead star's luminosity which may outshine momentarily the entire galaxy in which it resides. The explosion is produced by a catastrophic collapse of the iron core of a massive star or the collapse of a white dwarf after accreting enough mass from its companion to reach the Chandrasekhar limit. The first record of a supernova occurrence goes back to 185 CE. Subsequently, humans have witnessed across the centuries a series of such violent events that appear suddenly in the sky and illuminate the darkness of the night for several weeks or months. In the first part of this paper, we briefly describe the processes that lead to a supernova explosion. In the second part, we discuss historical supernovae as appearing in the records of human civilizations. In the third part, we highlight ancient records relating the sudden appearance of a supernova or a comet to the spread of epidemics in certain regions of the world.
\end{abstract}

\section{Keywords}

Supernovae Formation, Historical Supernovae, Epidemic Correlations

\section{Introduction}

Supernovae are violent stellar explosions. The physical process involved in generating supernovae has been the subject of intense theoretical and observational research during the past few decades. The amount of energy released in a typical supernova explosion is colossal and reaches the incredible level of about $10^{44}$ joules [1] [2]. The study of their spectra and light curves has been an efficient tool in identifying the nature of the processes producing the huge amount of energy released in these explosions. In a typical burst, the radiation emitted by a supernova 
may outshine, in a short period of time, the entire galaxy in which it resides.

The first record of historical supernovae went back to $185 \mathrm{CE}$ and was recorded by the Chinese [3]. Since then, several supernovae have been observed in our galaxy, the most luminous of which was SN 1006, which occurred in the southern constellation Lupus. The most recent supernova explosion in our galaxy occurred in 1604. For the past four centuries, our galaxy did not witness a supernova explosion. However, supernova sighting is limited by the observational capabilities and detection techniques. With the current set of satellites and Earth-based telescopes, it is probably difficult for a galactic supernova to escape detection.

In this paper, we review the different types of supernova and provide an estimate of their occurrences in the observed universe. We proceed then to review and discuss the records of historical supernovae in the Milky Way Galaxy and highlight the efforts to detect galactic supernovae remnants using new detection techniques. We next discuss certain records trying to link supernovae occurrences to the spread of epidemics in certain regions of the world. Observational data show that there has been confusion between the sudden appearance of certain ancient supernovae and comets. Modern theories regarding comets as a source of epidemics are discussed and are compared with ancient records in this respect. The role of modern spacecraft in studying the biological and other chemical and physical properties of comets is highlighted and discussed.

\section{Types of Supernovae}

The remnants of historical supernovae can provide a clue to the nature of the explosion that took place. The way stars come to a terminal stage of their evolution as a supernova is not unique and depends on the mass of the star and on its environment. There are two general types of supernova. The first type is of thermonuclear origin and is called Type I supernova. It is distinguished by the absence of any hydrogen line in its spectrum. This type is further subdivided into subtypes Ia, Ib, and Ic depending on the details of the spectrum. Type Ia is characterized by the presence of strong Si II lines at $615 \mathrm{~nm}$ and occurs when the mass of the dying star exceeds the Chandrasekhar limit of about 1.4 solar masses. Types Ib and Ic are designated according to the presence or absence of strong helium lines, respectively.

The second category of supernova is known as Type II supernova and is characterized by the presence of strong hydrogen lines in the spectrum. This type of supernova occurs in massive stars whose mass usually exceeds 8 solar masses. In these stars, the gravitational energy is strong enough to drive successive nuclear transformations in the inner layers of the star. Type Ib, Type Ic, and Type II supernovae are closely related, and they are produced by the collapse of a massive and evolved stellar core. These three types are referred to collectively as core collapse supernovae. Type II supernovae are also characterized by a rapid increase in luminosity, but their maximum brightness is typically 1.5 magnitudes 
dimmer than Type Ia supernovae. The peak is followed by a decrease in brightness of about six to eight magnitudes per year. These characteristics are very helpful, and they can be compared to available ancient records to identify the supernova's type, and consequently the nature of the explosion that took place.

\section{Historical Supernovae}

Our knowledge about historical supernovae relies entirely on ancient records left by human civilizations. These records are not always accurate, and to translate the texts into observational data has been one of the most challenging problems facing astronomers. Modern satellites and Earth-based observatories are the main tool to identify supernovae remnants left by the explosion. However, the celestial coordinates and age of these remnants need to match accurately the available ancient records.

SN 185: The oldest document of a supernova concerns an event that occurred in $185 \mathrm{CE}$ and was recorded by Chinese astronomers in the Book of Later Han as a "Guest Star" that remained visible in the night sky for about eight months [3]. It might also have been recorded in Roman literature [4]. The explosion was identified as the remnant RCW 86. It has a diameter of 85 light years and is 8000 light-years away in the southern constellation Circinus. NASA's Chandra X-Ray Observatory and the European Space Agency's XMM-Newton Observatory were used in combination to form a clear image of RCW 86. Infrared data provided by NASA's Spitzer Space Telescope and WISE (Wide Field Infrared Survey Explorer) observed clouds of dust radiating at very low temperatures, but warm enough in comparison to normal dust in our Milky Way Galaxy. X-ray and infrared data led astronomers to identify the explosion as a Type Ia supernova [5] [6], in which a white dwarf accretes enough mass from its companion star to exceed the Chandrasekhar mass limit. Also, scientists need to solve one of the mysteries surrounding the remnant, namely, how it got so large in such a short time. This could be explained by assuming that a huge cavity was cleared out by a violent wind produced by the white dwarf prior to the explosion. This cavity is a very low-density region where the remnant was able to expand at a much faster rate than it would have otherwise.

SN 386: This is a transient astronomical event which appeared as a "Guest Star" in the constellation Sagittarius and was recorded by Chinese astronomers almost 200 years after the first record of $185 \mathrm{CE}$ [7]. Earlier reports associate SN 386 with the supernova remnant SNR G11.2-0.3 [8]. However, recent studies show that this theory is not valid [9] [10]. In fact, a measured mean expansion rate of the SNR G11.2-0.3 remnant shell is $(0.0277 \pm 0.0180) \%$ per year, and the actual diameter of the remnant now is about 9.8 ly, suggesting an age of $1900 \pm$ 500 years [9]. Recent radio observations have shown that the remnant is 14,000 to 23,000 ly away [9]. A serious reason to reject the association of SNR G11.2-0.3 with SN 386 is the very high absorption of light between the source and the Earth, which is estimated from infrared absorption at about 16 magnitudes. This 
suggests that the exploding star would not have been visible to the naked eye. Further evidence of the failure to associate SNR G11.2-0.3 with SN 386 arises from the characteristics of the pulsar PSR J1811-1926 residing at the core of the remnant [11]. Recent measurements of the rotational velocity spin down rate and radio observations of PSR J1811-1926 indicate a much older age ranging between 20,000 to 23,000 years [9]. If confirmed, this would clearly discount the SNR G11.2-0.3 remnant as being associated with SN 386. There is a clear contradiction between the age determined by the expansion rate of the supernova remnant and the pulsar at its core as determined by its spin-down rate. As a conclusion, the mystery of SN 386 remains unsolved. An alternative explanation is that the event reported by the Chinese refers to a passing comet. However, this suggestion has also its drawbacks since comets will not appear suddenly as "Guest Stars".

SN 393: The Chinese recorded the appearance of a "Guest Star" in 393 CE in the constellation Scorpios. The guest star remained visible for about eight months before fading and becoming invisible [12]. Prior to 1975, the Chinese record of $393 \mathrm{CE}$ was interpreted as a likely bright nova with a secondary minimum [13]. At the time, there were only seven possible supernova remnant candidates in the region of the sky where SN 393 was observed. Assuming a maximum magnitude of -1 for an event occurring close to 33,000 ly away, this immediately rules out four possible candidates [13]. Another discounted candidate is the G350.0-18 remnant whose expansion rate suggests that the supernova occurred some 8000 years ago. The remaining two supernova remnants in this region of the sky are G348.5 + 0.1 and G348.7 + 0.3; both are at the required distance of 33,000 ly and both have an estimated age of 1500 years [13]. If confirmed, it seems unlikely that such supernovae would be visible to the naked eye for eight months, especially because they occurred close to a particularly dusty region in the galactic plane [14]. Fortunately, the ROSAT All Sky Survey discovered in 1996 another nearby supernova remnant, RX J1713.7-3946, which was suggested as a better match for SN 393 [15]. It was suggested in 1996 that the remnant was associated with the $\mathrm{H}_{\text {II }}$ region G347.611 + 0.204 with an estimated distance of 20,000 ly. However, in 2003, when examining the interactions between a nearby molecular cloud and the expanding remnant, it was found that the distance is only 3000 ly. Furthermore, in 2004, measurements of the degree of X-ray and neutral hydrogen absorption by intervening matter between the Earth and the remnant, confirmed this distance. Supernova remnant RX J1713.7-3946 is consistent with a Type II or Type Ib supernova with a progenitor having a mass of 15 solar masses [16].

SN 1006: This is the brightest ever observed supernova recorded in history. It appeared as a "Guest Star" between the $30^{\text {th }}$ of April and the $1^{\text {st }}$ of May 1006 in the constellation Lupus and reached an estimated visual magnitude of -7.5 [17]. This brightness exceeded roughly sixteen times the brightness of Venus. The appearance of the "Guest Star" was recorded in Egypt, Iraq, China, Europe, Japan [18] [19], Yemen [20], and possibly by North American petroglyphs [21]. 
The distance to SN 1006 is estimated to be 7200 ly.

Egyptian astrologer and astronomer Ali Bin Ridwan described the event in a commentary on Ptolemy's Tetrabiblos as: “The spectacle was a large circular body, 2.5 to 3 times as large as Venus. The sky was shining because of its light. The intensity of its light was a little more than a quarter that of Moon light" [18]. It is interesting to note that certain astrologers interpreted the event as a portent of plague and famine [18]. This belief was deeply rooted in various cultures worldwide at the time, as described later in Section 5.

In Europe, the event was recorded in the annals of the Abbey of Saint Gall in Switzerland [18]. Monks at St. Gall described the event as: "In a wonderful manner this was sometimes contracted, sometimes diffused, and moreover sometimes extinguished. It was seen likewise for three months in the inmost limits of the South, beyond all constellations which are seen in the sky" [22]. This record is often taken as an evidence of a Type Ia supernova. The "Guest Star" was described as bright enough to cast shadows and it was certainly seen during daylight hours for some time [23].

Songshi, the official historian of the Song Dynasty described the star as appearing on $1^{\text {st }}$ of May 1006 in the constellation Di, east of Lupus, as shining so brightly that objects on the ground could be seen at night. In December of the same year, it was again sighted in the constellation $\mathrm{Di}$, and was described by the Chinese astrologer Zhou Koming as an auspicious star, yellow in color and brilliant, that would bring great prosperity to the states over which it appeared [18]. The above description suggests that there were two distinct phases in the early evolution of supernova SN 1006. The first phase extended for a period of three months where the brightness reached its maximum. After this period, the brightness diminished, and then returned for a period of about eighteen months. There are also reports of this supernova in a petroglyph by the Hohokam in White Tank Mountain Regional Park in Arizona which was interpreted as the first known North American representation of a supernova [21]. Moreover, a recently found document discovered on the $25^{\text {th }}$ of August 2015 suggests that observers in Yemen may have seen SN 1006 on the $17^{\text {th }}$ of April 1006, two weeks before its previously assumed earliest observation [20].

The SN 1006 supernova remnant was first identified in 1965. Doug Milne and Frank Gardner demonstrated an association of the known radio source PKS 1459-41 with SN 1006 [24]. The remnant (SNR G327.6+14.6) is a circular shell located near the star Beta Lupi [25]. X-ray and optical emission from this remnant have been detected. In 2010, the H.E.S.S. gamma-ray observatory announced the detection of very high energy gamma-ray emissions from the remnant [25]. A signal of the SN 1006 outburst was found in the nitrate deposits in Antarctic ice [26]. No associated neutron star or black hole has been found, which is the expected situation for the remnant of a Type Ia supernova [27]. A survey in 2012 searching for any surviving companions of the SN 1006 progenitor failed to find anything [28]. It was suggested that SN 1006 was most likely a 
double degenerate progenitor, that is, it was produced by the merging of two white dwarf stars. This model is in line with the observed two phases of the brightness of the supernova. The two progenitors burst out successively giving rise to two peaks of brightness. The estimated distance of the remnant SNR G327.6 + 14.6 is about 7432 ly.

SN 1054: Probably this is the most famous supernova in history. It was first observed on the $4^{\text {th }}$ of July 1054 . The event was recorded by the Arabs, Chinese, Japanese, and probably by the Europeans and North Americans as evidenced by a pictograph associated with Ancestral Puebloan culture found near the Peñasco Blanco site in New Mexico. There are also a few proposed, but doubtful, observations from European sources recorded in the $15^{\text {th }}$ century. The supernova remained visible for two years. The supernova remnant was photographed by the Hubble Space Telescope. The SN 1054 remnant is located on the sky near Zeta Tauri and is known as the Crab Nebula. It was identified as the remnant of SN 1054 in the years between 1921 and 1942. At the core of the remnant lies a pulsar. Carl Otto Lampland announced in 1921 that he had seen a structure in the Crab Nebula [29]. In 1928, Edwin Hubble was the first to notice the changing aspect of the Crab Nebula, due to its growing size, which he attributed to a stellar explosion and thus confirmed that the nebula is the remnant of a supernova. From the change in size, Edwin Hubble calculated the date of explosion and found that it had occurred about nine centuries ago. However, Hubble's comments remained obscure since the physical phenomenon of the explosion was not known at the time. Eleven years later, Walter Baade and Fritz Zwicky highlighted the fact that supernovae are very bright phenomena [30]. An explanation of their nature was suggested by Zwicky [31]. Nicholas Mayall was the first to propose that the event of 1054 was actually a supernova [32]. This deduction was subsequently refined by Mayall and Jan Oort in 1942.

Chinese sightings: In Chinese records, the "Guest Star" of 1054 occurred during the reign of Renzong of the Sing dynasty (960-1279). The event was recorded in Chinese documents in the first year of the Zhihe era, corresponding to the year $1054 \mathrm{CE}$. Many Chinese documents identify the position and date of the appearance of the "Guest Star". Three Chinese documents indicate that the guest star was located close to the South-East of Tianguan (Zeta Tauri). The duration of the supernova is explicitly mentioned in Chinese records. The Song Shi is the official annals of the Song dynasty, and it gives a total period of visibility of 642 days; according to the Song Huiyo record, daylight visibility of the event lasted for 23 days.

Japanese sightings: In Japan, the oldest and most detailed record is the Meigetsuki, the diary of Fujiwara no Teika (1162-1241). The Meigetsuki places the event in the fourth lunar moon, one month earlier than the Chinese texts. This difference is usually attributed to an error in the lunar months in the Japanese document. The location of the "Guest Star" was in the direction of the East, and it seemed to emerge from the Tianguan star. 
Arabian sightings: In Arabian records, the sighting of SN 1054 appeared in the Book Uyon Al Anbaa fi Tabaquat Al Atibaa. This is a detailed bibliography of physicians in the Islamic World compiled by Ibn Abi Usaibi'a (1194-1270) in the middle of the thirteenth century. In this book, the testimony was given by Ibn Batlan, who stated: "One of the famous epidemics of our time occurred when a spectacular star appeared in Gemini, of the year 446 (of the Muslim Calendar). In the autumn of that year, fourteen thousand people were buried in Constantinople. Thereafter, in the middle of the summer of 447, the majority of Fostat (Cairo) people and all foreigners died". Ibn Batlan continues: "While the spectacular star appeared in the sign of Gemini, it caused the epidemic of the Fostat with the Nile being low, when it appeared in 445" [33]. The year 446 in the Muslim Calendar corresponds to the year 1054 CE. The exact date of the event in 446 is difficult to determine, but the reference in the text to the level of the Nile indicates that it occurred during the period preceding the annual flood, which happens during summer, in good agreement with the Chinese records.

European sightings: Since 1980, several European documents have been identified as potential observations of supernova SN 1054 [34] [35] [36] [37] [38]. Most of these documents are not precise, and they are not convincing from an astronomical point of view. It seems difficult to interpret most of them as related to a possible supernova event. The lack of accounts in European chronicles about SN 1054 raised several questions. In fact, SN 1054 occurred only 48 years after the famous SN 1006 which was recorded in a substantial number of European documents. In 1999, George W. Collins and coworkers [34] defended the plausibility of the European sighting of SN 1054, arguing that the records indicate that European sightings predate Chinese and Japanese records by more than two months. These authors criticize the Chinese records, particularly the problem associated with the position of the supernova with respect to the Zeta Tauri star. They also surmise that the correct year reported by Ibn Batlan is AH 445 (which corresponds to: 23 April 1053 - 11 April 1054) rather than 446 (which corresponds to: 12 April 1054 - 1 April 1055). The publication by Collins et al. [34] was highly criticized by Stephenson and Green [39]. These authors emphasize that the problem with Chinese and Japanese documents can be easily solved philosophically and need not be interpreted in terms of unreliability in the Chinese and Japanese observations [39]. They argue that the consensus established by 1995 is that the European documents do not provide an interpretation of the sighting of SN 1054 [40]. The idea of Collins et al. [34] was reviewed with some enthusiasm in the magazine Ciel \& Espace, but it has not received much support due to its criticism by Stephenson and Green [41].

North American Petroglyphs: The SN 1054 event appeared in two Native American paintings. These paintings show a crescent moon located next to a circle that could represent a star [42]. It was suggested that this configuration represents a conjunction between the moon and the supernova. This is made possible by the fact that, seen from the Earth, the supernova occurred in the path 
of the ecliptic. This suggestion is in line with the dating in these paintings. More precisely, on the morning of July $5^{\text {th }}, 1054$, the moon was located in the immediate proximity of the supernova, as depicted in these paintings. However, this interpretation cannot be confirmed, since the dating of the paintings is extremely imprecise, and only one of the paintings shows the crescent moon. Another, more precise document is related to the Anasazi at the Chaco Canyon site in New Mexico. It shows a hand below which there is a crescent moon facing a star at the bottom left. On the wall underneath the petroglyph there is a drawing which could be the core and tail of a comet. This could be the passing of Halley's Comet in 1066. Although plausible, this interpretation is impossible to confirm and does not explain why it was the supernova SN 1054 that was represented.

SN 1181: Chinese and Japanese astronomers recorded the SN 1181 supernova in eight separate texts. The event took place on August 4 and August 6, 1181. It is one of only eight recorded supernovae that were observable with the naked eye. It remained visible for about 185 days in the direction of the constellation Cassiopeia.

The remnant of SN 1181 is believed to be associated with the X-ray pulsar $\mathrm{J} 0205+6449$ (also known as 3C58). The pulsar's rotational period is 15 rotations per second, and it is still rotating as quickly as it did when it first formed [43]. This is in contrast with the Crab Nebula pulsar (SN 1054 remnant), which has lost two thirds of its rotational velocity in essentially the same time span [44]. The surprise came from recent radio surveys of 3C58 that indicate that this supernova remnant is much older, and therefore cannot be associated with $\mathrm{SN}$ 1181. The remnant of SN 1181 is still to be identified.

SN 1572: A supernova that appeared in early November 1572 in the constellation Cassiopeia was discovered independently by many individuals [45]. SN 1572 is considered one of the most important events in the history of astronomy. Tycho Brahe produced an extensive work describing the observation of a "Guest Star": "De nova et nullius aevi memoria prius" (concerning the star, new and never before seen in the life memory of anyone). The work was published in 1573 with reprints overseen by Johannes Kepler in 1602 and 1610. These reprints contained both Tycho Brahe's own observation and the analysis of sightings by many other observers.

In England, John Aubrey recorded in his memoranda the impact of the event on Queen Elizabeth [46]. She summoned the mathematician and astrologer Thomas Allen asking his advice about the new star that appeared in Cassiopeia. Thomas gave his judgment very learnedly. In the Ming dynasty in China, the appearance of the "Guest Star" was an issue of discussion between the young Wanli Emperor and Zhang Juzhang. According to the cosmological tradition, the emperor was warned to consider his misbehavior since the appearance of the new star was interpreted as an evil omen [47]. More reliable contemporary reports mention a star that burst forth sometime between November $2^{\text {nd }}$ and $6^{\text {th }}$ in 1572, driving the star to rival Venus in brightness [48]. The supernova remained visible to the naked eye well into 1574 before gradually fading and disappearing 
from view [48]. In Yemen, the supernova was observed by Isa Sharaf-Eldine, and was published in his book Rawh El-Rooh [49].

Considerable efforts were deployed to search for the supernova remnant associated with SN 1572, but these efforts were not conclusive until 1952, when Hanbury Prawn and Cyril Hazard from Jodrell Bank Observatory detected a radio signal at $158.5 \mathrm{MHz}$. The remnant was identified as the object 3C10 appearing in the third Cambridge list, and another designation of SN 1572 is G120.7 + 2.1, but most authors simply refer to it as the "Tycho supernova remnant". The visible counterpart of SN 1572 was discovered in the 1960s, using the Palomar Mountain Telescope, as a faint nebula. The supernova was confirmed as Type Ia [48]. Recent studies indicate a rate of expansion lower than $5000 \mathrm{~km} / \mathrm{s}$ [50]. In October 2004, the discovery of a G2 star, like our Sun, was reported as a companion star that contributed mass to the exploding white dwarf of SN 1572. The star was named Tyhco G. It has a velocity exceeding four times the mean velocities of neighboring stars. This discovery has been challenged recently since the star is far away from the center of the explosion and does not show rotation, which is expected for a companion star [51].

SN 1604: This is also known as Kepler's supernova. This is the last supernova observed in our galaxy. It appeared in the constellation Ophiuchus in 1604. SN 1604 was brighter than any other star in the night sky, with an apparent magnitude of -2.5. It remained visible during the day for over three weeks.

The first record of SN 1604 was by Lodovico delle Colombe in northern Italy on the $9^{\text {th }}$ of October 1604 [46]. Johannes Kepler started his observation of the event on the $17^{\text {th }}$ of October while working at the imperial court in Prague for Emperor Rudolf [46]. It was subsequently named after him, even though he was not the first to observe the event. The supernova was also recorded by the Arabian astronomer Isa Sharaf-Eldine [49], and thus he is the only known astronomer who witnessed the appearance of two supernovae. The supernova was also observed by Chinese and Korean sources [47].

Table 1 provides a summary of the recorded historical supernovae that

Table 1. Recorded historical supernova events.

\begin{tabular}{|c|c|c|c|c|c|}
\hline Observation & Type & Magnitude & Constellation & Date (month) & Year \\
\hline Chinese & Ia & - & Circinus \& Centaurus & - & $185 \mathrm{CE}$ \\
\hline Chinese & - & - & Sagittarius & - & $386 \mathrm{CE}$ \\
\hline Chinese & $\mathrm{II}-\mathrm{Ib}$ & -1 & Scorpius & - & $393 \mathrm{CE}$ \\
\hline Arabs, Chinese, Japanese, Europeans & Ia & -7.5 & Lupus & 30 April & $1006 \mathrm{CE}$ \\
\hline North Americans, Arabs, Chinese & II & -6 & Taurus & 4 July & $1054 \mathrm{CE}$ \\
\hline Chinese and Japanese & - & $-1 ?$ & Cassiopeia & 6 August & $1181 \mathrm{CE}$ \\
\hline Chinese, Japanese, Arabs, Europeans, Tycho Brahe & Ia & -4 & Cassiopeia & 6 November & $1572 \mathrm{CE}$ \\
\hline Europeans, Arabs, Kepler & Ia & -2.25 to -2.5 & Ophiuchus & 9 October & $1604 \mathrm{CE}$ \\
\hline
\end{tabular}


were discussed and reviewed in this paper.

\section{Unrecorded Milky Way Galaxy Supernovae}

For the past four centuries, no supernova was reported in our galaxy, though many others have been observed in other galaxies, the most important of which is the SN 1986 supernova which appeared in the Large Magellanic Cloud and was visible to the naked eye. There is evidence for two Milky Way Galaxy supernovae that occurred in 1680 and 1870, however, they were not recorded by any source. These are Cassiopeia A [52] and G1.9 + 0.3 [53], respectively. Cassiopeia A occurred in the constellation Cassiopeia and is a core collapse supernova of Type IIb [54], whereas G1.9 + 0.3 occurred in the constellation Sagittarius and was triggered by the merger of two white dwarfs [53]. The absence of historical records for either is probably due to absorption by interstellar dust which made them fainter than usual and thus difficult to observe. Other unconfirmed supernova events may have been observed in $369 \mathrm{CE}, 386 \mathrm{CE}, 437 \mathrm{CE}$, $827 \mathrm{CE}$, and $902 \mathrm{CE}[55]$.

\section{Supernovae, Comets, and the Epidemic Claim}

Some Arab and European documents relate the appearance of "Guest Stars" to the spread of epidemics in certain regions in the world. The most famous document of the kind is the book by Ibn Abi Usaibi'a Uyon Al Anbaa fi Tabaquat Al Atibaa, already mentioned in relation to SN 1054. Ibn Batlan described in the text the spread of epidemics in several regions in the Middle East concurrently with the appearance of a "Guest Star". Scientifically, the relation between the explosion of a supernova and the spread of an epidemic is an idea that lacks any scientific merit. For the case of the SN 1054 supernova, light takes 6500 years to arrive the Earth. Any biological material from the exploding star environment cannot be ejected with velocities comparable to the speed of light, and consequently it will arrive on Earth hundreds to thousands of years later. Furthermore, biological materials cannot withstand the high temperatures caused by the explosion. In the second part of the document, Ibn Batlan stated: "Thus Ptolemy's prediction becomes true: Woe to the people of Egypt when one of the comets appears threateningly in Gemini! Then Saturn descended into the sign of Cancer, ... Famine and epidemics abandoned". In the above paragraph, Ibn Batlan quotes Ptolemy to emphasize the relation between comets and the spread of epidemics. It seems that there was confusion at that time between supernovae and comets. The relation between comets and the spread of epidemics was discussed thoroughly (in modern times) by Hoyle and Wickramasinghe in their book Evolution from Space [56]. They argue that the chance of obtaining the required set of enzymes for even the simplest cell without panspermia is one in $10^{40000}$. This means that the chance is basically zero. Hoyle and Wickramasinghe advance the idea that comets are the source of panspermia, and they attribute the diseases on the Earth to extraterrestrial origins, including the 1918 flu pan- 
demic, and certain outbreaks of polio and mad cow diseases. For the 1918 flu pandemic they hypothesized that cometary dust brought the virus to the Earth simultaneously at multiple locations. This view was almost universally dismissed by experts of this pandemic. The possibility that comets may carry biological materials has been considered seriously in recent years by various space agencies. This idea was the main reason for sending space probes to visit comets and investigate the possibility of detecting biological materials on their surfaces. Among the missions to visit comets are Vega 1 and 2 (1986), Giotto (1992), Deep Space (2001), Stardust (2004), Deep Impact (2005), Ulysses (2007), and Rosetta (2016). The outcome of these missions was not conclusive.

\section{Conclusion}

Historical supernovae research studies have flourished in modern times. The problem of identifying the location of the corresponding supernova remnant has been one of the most challenging endeavors facing astronomers today. The challenge has been to extract reasonable information from (at times) poorly written historical records. This is the case, for example, with SN 386. Other problems are associated with the contradictory Chinese and Japanese records, especially in the case of SN 1054. Compared to other nations, the Chinese were the most diligent in recording the appearance of "Guest Stars". In the Arab world, several dispersed records revealing the sightings of "Guest Stars" have been found. Ibn Radwan from Egypt, Ibn Batlan from Iraq, and Isa Sharaf-Eldine from Yemen are the main names appearing in Arabic historical records of supernovae sightings. The latter is the only astronomer who has sighted two supernovae in a lifetime. A disturbing Arabic record written by Ibn Batlan tried to associate the appearance of the SN 1054 supernova with the spread of epidemics in certain regions in the Middle East. We have shown that such a claim is devoid of any scientific merit. Two newly discovered historical supernovae in our galaxy escaped detection, most probably because of the intervening interstellar dust. It is interesting to note that most recorded supernovae are of Type Ia. With the advent of more advanced observational facilities, it might be possible in the near future to precisely pinpoint the location of some doubtful historical supernovae remnants.

\section{Conflicts of Interest}

The authors declare no conflicts of interest regarding the publication of this paper.

\section{References}

[1] Woosley, S. and Janka, T.H. (2005) The Physics of Core-Collapse Supernovae. https://doi.org/10.1038/nphys172

[2] Khokhlov, A.M., et al. (1999) Jet-Induced Explosions of Core Collapse Supernovae. The Astrophysical Journal, 524, L107. https://doi.org/10.1086/312305 
[3] Zhao, F.Y., Strom, R.G. and Jiang, S.Y. (2006) The Guest Star of AD185 Must Have Been a Supernova. Chinese Journal of Astronomy and Astrophysics, 6, 635-640. https://doi.org/10.1088/1009-9271/6/5/17

[4] Stothers, R. (1977) Is the Supernova of A.D. 185 Recorded in Ancient Roman Literature? Isis, 68, 443-447. https://doi.org/10.1086/351822

[5] NASA, JPL (2011) NASA Telescopes Help Solve Ancient Supernova Mystery. https://www.nasa.gov/mission pages/spitzer/news/spitzer20111024.html

[6] Williams, B.J., et al. (2011) RCW 86: A Type Ia Supernova in a Wind-Blown Bubble. The Astrophysical Journal, 741, 96. https://doi.org/10.1088/0004-637X/741/2/96

[7] Clark, D.H. and Stephenson, F.R. (1976) Which Historical New Stars Were Supernovae? The Quarterly Journal of the Royal Astronomical Society, 17, 290.

[8] Mizuno, D.R., et al. (2010) A Catalog of MIPSGAL Disk and Ring Sources. The Astronomical Journal, 139, 1542-1552. https://doi.org/10.1088/0004-6256/139/4/1542

[9] Borkowski, K.J., Reynolds, S.P. and Roberts, M.S.E. (2016) G11.2-0.3: The Young Remnant of a Stripped-Envelope Supernova. The Astrophysical Journal, 819, 160. https://doi.org/10.3847/0004-637X/819/2/160

[10] Kaspi, V.M., et al. (2001) Chandra X-Ray Observations of G11.2-0.3: Implications for Pulsar Ages. The Astrophysical Journal, 560, 372.

https://doi.org/10.1086/322515

[11] Tam, C. and Roberts, M.S.E. (2003) The Expansion of G11.2-0.3, a Radio Composite Supernova Remnant. The Astrophysical Journal Letters, 598, L27-L30. https://doi.org/10.1086/380557

[12] Clark, D.H. and Stephenson, F.R. (1975) The Remnants of the Supernovae of AD 185 and AD 393. The Observatory, 95, 190-195.

[13] Clark, D.H. and Stephenson, F.R. (1978) Application of Early Astronomical Records. Institute of Physics Publishing, London.

[14] Wang, Z.-R. (2005) The Impact of Historical Chinese Astronomical Records. Astrophysics and Space Science, 305, 207-210.

https://doi.org/10.1007/s10509-006-9187-8

[15] Wang, Z.-R., Qu, Q.Y. and Chen, Y. (1998) The AD 393 Guest Star: The SNR RX 51713.7-3946. The Hot Universe. In: Koyama, K., Kitamoto, S. and Itoh, M., Eds., Proceedings of IAU Symposium \#188, Kluwer Academic, Dordrecht, 262. https://doi.org/10.1007/978-94-011-4970-9 75

[16] Berezhko, E.G. and Völk, H.J. (2010) Nonthermal and Thermal Emission from the Supernova Remnant RX J1713.7-3946. Astronomy and Astrophysics, 511, A34. https://doi.org/10.1051/0004-6361/200913312

[17] National Optical Astronomy Observatory (2003) Astronomers Peg Brightness of History's Brightest Star. Press Release.

https://noirlab.edu/public/news/noao0305

[18] Murdin, P. and Murdin, L. (1985) Supernovae. Cambridge University Press, Cambridge, 14-16.

[19] Burnham, R. (1978) Celestial Handbook. Dover, New York, 1117-1122.

[20] Rada, W. and Heuhaeuser, R. (2015) Supernova SN 1006 in Two Historic Yemeni Reports. Astronomische Nachrichten, 336, 249-257. https://doi.org/10.1002/asna.201412152

[21] Than, K. (2006) Ancient Rock Art Depicts Exploding Star. Space.com. https://www.space.com/2458-ancient-rock-art-depicts-exploding-star.html 
[22] Goldstein, B.R. (1965) Evidence for a Supernova of A.D. 1006. The Astronomical Journal, 70,105-114. https://doi.org/10.1086/109679

[23] Winkler, P.F., et al. (2002) The SN 1006 Remnant: Optical Proper Motions, Deep Imaging, Distance, and Brightness at Maximum. https://doi.org/10.1086/345985

[24] Gardner, F.F. and Milne, D.K. (1965) The Supernova of A.D. 1006. The Astronomical Journal, 70, 754. https://doi.org/10.1086/109813

[25] Green, D.A. (2014) A Catalogue of 294 Galactic Supernova Remnants. Bulletin of the Astronomical Society of India, 42, 47-58.

[26] Acero, F., et al. (2010). First Detection of VHE $\gamma$-Rays from SN 1006 by HESS. Astronomy and Astrophysics, 516, A62. https://doi.org/10.1051/0004-6361/200913916

[27] Wheeler, J.C. (2000) Cosmic Catastrophes: Supernovae, Gamma-Ray Bursts, and Adventures in Hyperspace. Cambridge University Press, Cambridge.

[28] González Hernández, J.I., et al. (2012) No Surviving Evolved Companions of the Progenitor of SN 1006. Nature, 489, 533-536. https://doi.org/10.1038/nature11447

[29] Lampland, C.O. (1921) Observed Changes in the Structure of the "Crab" Nebula. Publications of the Astronomical Society of the Pacific, 33, 79-84. https://doi.org/10.1086/123039

[30] Baade, W. and Zwicky, F. (1934) On Supernovae. Proceedings of the National Academy of Sciences, 20, 254-259. https://doi.org/10.1073/pnas.20.5.254

[31] Zwicky, F. (1938) On Collapsed Neutron Stars. The Astrophysical Journal, 88, 522-525. https://doi.org/10.1086/144003

[32] Mayall, N.U. (1939) The Crab Nebula, a Probable Supernova. Astronomical Society of the Pacific Leaflets, 3, 145-154.

[33] Brill (n.d.) A Literary History of Medicine. Oxford Center for Islamic Studies, Oxford.

https://www.oxcis.ac.uk/news/literary-history-of-medicine-the-uyun-al-anba-fi-tab aqat-al-atibba-of-ibn-abi-usaybiah

[34] Collins, G.W., Claspy, W.P. and Martin, J.C. (1999) A Reinterpretation of Historical References to the Supernova of A.D. 1054. Publications of the Astronomical Society of the Pacific, 111, 871-880. https://doi.org/10.1086/316401

[35] Dall'Olmo, U. (1980) Latin Terminology Relating to Aurorae, Comets, Meteors and Novae. Journal for the History of Astronomy, 11, 10-27. https://doi.org/10.1177/002182868001100103

[36] Williams, L.P. (1981) The Supernova of 1054: A Medieval Mystery. In: Woolf, H., Ed., The Analytic Spirit. Essays in the History of Science in Honor of Henry Guerlac, Cornell University Press, Ithaca, 329-349.

[37] Guidoboni, E., Marmo, C. and Polcaro, V.F. (1994) Do We Need to Redate the Birth of the Crab Nebula? Memorie della Società Astronomia Italiana, 65, 623-637.

[38] McCarthy, D. and Breen, A. (1997) An Evaluation of Astronomical Observations in the Irish Annals. Vistas in Astronomy, 41, 117-138. https://doi.org/10.1016/S0083-6656(96)00052-9

[39] Stephenson, F.R. and Green, D.A. (2003) Was the Supernova of AD 1054 Reported in European History? Journal of Astronomical History and Heritage, 6, 46-52.

[40] Breen, A. and McCarthy, D. (1995) A Re-Evaluation of the Eastern and Western Records of the Supernova of 1054. Vistas in Astronomy, 39, 363-379. https://doi.org/10.1016/0083-6656(95)96619-S

[41] Stephenson, F.R. and Green, D.A. (2003) The Historical Supernovae. 
[42] Miller, W.C. (1955) Two Prehistoric Drawings of Possible Astronomical Significance. Astronomical Society of the Pacific Leaflets, 7, 105-112.

[43] Panagia, N. and Weiler, K.W. (1980) The Absolute Magnitude and the Type Classification of SN 1181 Equals 3C58. Astronomy and Astrophysics, 82, 389-391.

[44] Galas, C.M.F., Tuohy, I.R. and Garmire, G.P. (1980) Soft X-Ray Observations of the Supernova Remnants HB 3 and 3C58. The Astrophysical Journal Letters, 236, L13-L16. https://doi.org/10.1086/183188

[45] Science Daily (2008) Blast from the Past: Astronomers Resurrect 16th-Century Supernova. https://www.sciencedaily.com/releases/2008/12/081203133809.htm

[46] Dick, O.L. (1949) Aubrey's Brief Lives. Edited from the Original Manuscript "Thomas Allen". 5.

[47] Huang, R. (1981) 1587, a Year of No Significance. Yale University Press, London.

[48] Krause, O., et al. (2008) Tycho Brahe's 1572 Supernova as a Standard Type Ia as Revealed by Its Light-Echo Spectrum. Nature, 456, 617-619. https://doi.org/10.1038/nature07608

[49] Sharaf-Eldine, I. (n.d.) Rawh El-Rooh. Arabic Manuscript (Not Published).

[50] Hayato, A., et al. (2010) Expansion Velocity of Ejecta in Tycho's Supernova Remnant Measured by Doppler Broadened X-Ray Line Emission. The Astrophysical Journal, 725, 894-903. https://doi.org/10.1088/0004-637X/725/1/894

[51] Kerzendorf, W.E., et al. (2013) A High-Resolution Spectroscopic Search for the Remaining Donor for Tycho's Supernova. The Astrophysical Journal, 774, 99. https://doi.org/10.1088/0004-637X/774/2/99

[52] Fesen, R.A., et al. (2006) The Expansion Asymmetry and Age of the Cassiopeia A Supernova Remnant. The Astrophysical Journal, 645, 283-292. https://doi.org/10.1086/504254

[53] NASA, Chandra (2008) G1.9+0.3: Discovery of Most Recent Supernova in Our Galaxy. https://chandra.harvard.edu/photo/2008/g19

[54] Krause, O., et al. (2008) The Cassiopeia A Supernova Was of Type IIb. Science, 320, 1195-1197. https://doi.org/10.1126/science. 1155788

[55] Clark, D.H. and Stephenson, F.R. (1981) The Historical Supernovae. In: Supernovae: A Survey of Current Research, D. Reidel Publishing Co., Dordrecht, 355-370. https://doi.org/10.1007/978-94-009-7876-8 22

[56] Hoyle, F. and Wickramasinghe, C. (1984) Evolution from Space. Simon \& Schuster, New York. 